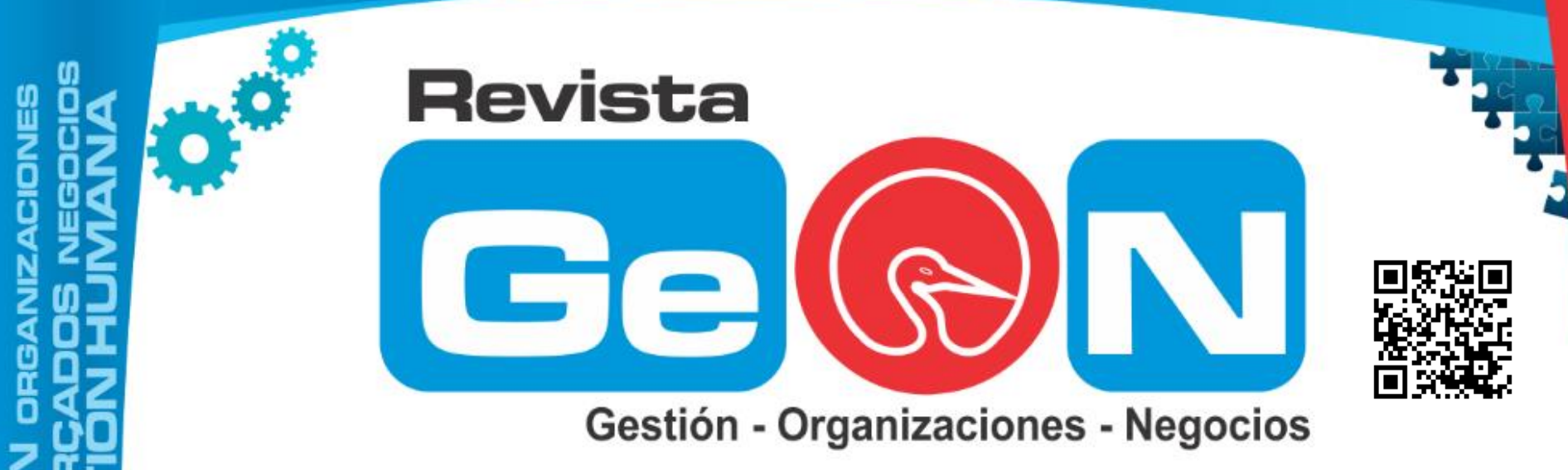

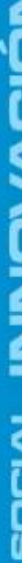

(1)

ISEN 204E - 3970

Volumen 11 Número 0 Diciembre Jullo 14

Revista Electrónica de la Facultad de Ciencias Económicas de la Universidad de los Llanos

$4 \leq 1$

$3<$

$2 \frac{1}{2} \frac{1}{4}$
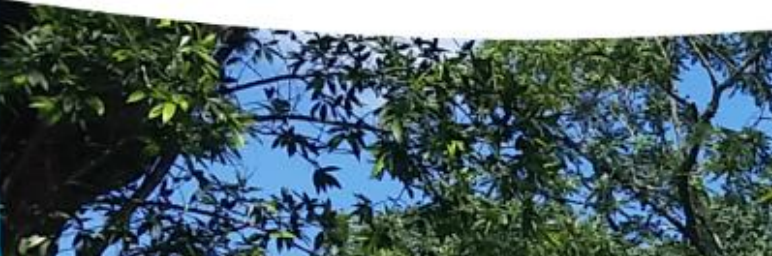

(1)

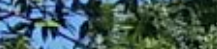

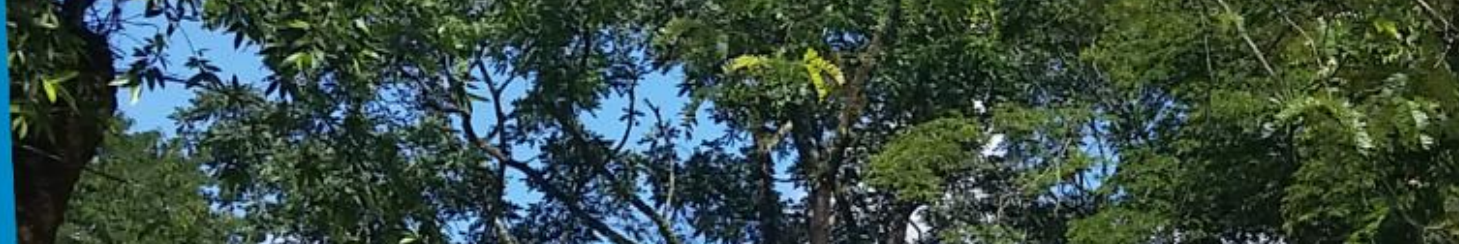

다

(11) को

$2 \frac{1}{0}$

i c

iii $\frac{2}{11}$ if

in

402

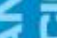

40

20 III

iㅣㄴ

12

10

10

ii 1

U1

$2 \frac{1}{2}$

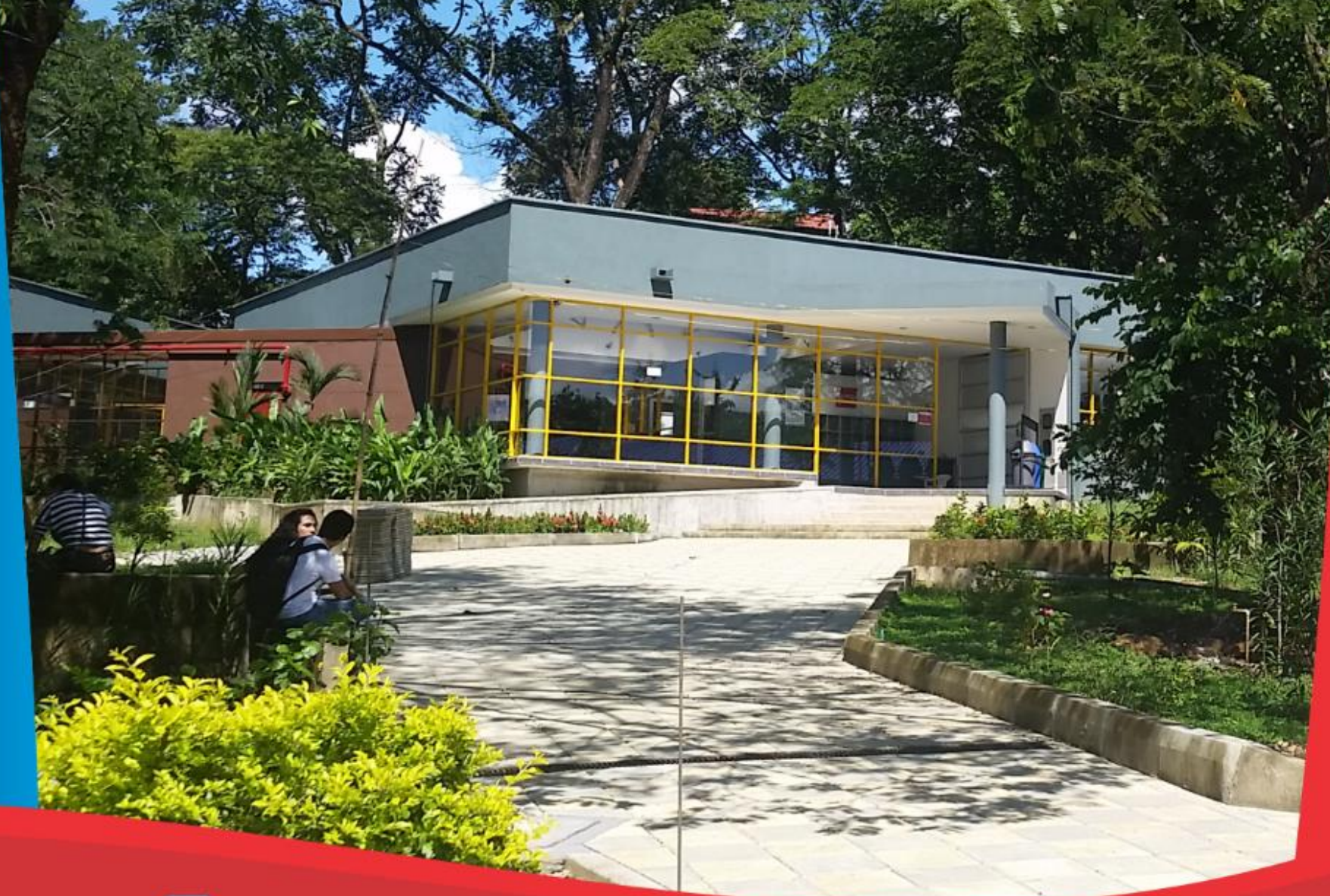

I/revistaGEON @RevistaGeon

http://revistageon.unillanos.edu.co 


\section{Análisis de la deserción y la repitencia académica en el programa de Administración de Empresas de la Universidad de los Llanos del 2008 al 2012 - Por Julieth sierra}

Julieth Andrea Sierra Tobón ${ }^{2}$

Administradora de Empresas, Especialista en Gestión

para el Desarrollo Empresarial (Actualmente)

@ sierrajulieth

\section{RESUMEN}

El presente artículo tiene como objetivo principal el ofrecer una síntesis del estudio que hiciese la autora sobre la deserción y la repitencia en el programa de Administración de Empresas de la Universidad de los Llanos durante el periodo comprendido entre los años 2008 y 2012, así como de su propuesta para afrontar dichos fenómenos y mejorar los índices de retención y rendimiento académico de los estudiantes de dicho programa. Para lograr tal cometido se ha dividido el escrito en dos partes: En la primera se expone, de manera resumida, el marco conceptual y la metodología utilizada en el estudio; en la segunda se presenta una síntesis del diagnóstico de la deserción y la repitencia académica en el periodo seleccionado.

Palabras clave: Deserción, repitencia, retención académica, SPADIES (Sistema de Prevención de la Educación Superior), SIA (Sistema de Información Académica de la Universidad de los Llanos).

\section{ABSTRACT}

This article's main objective is to offer a synthesis of the study that would make the author on dropout and repetition in the Business Administration program at the University of the Llanos during the period between 2008 and 2012, as well as its proposal to address these phenomena and improve retention rates and academic performance of students in the program. To accomplish that task has been divided writing into two parts: The first part presents, in summary, the conceptual framework and methodology used in the study; in the second a summary of the diagnosis of dropout and repetition in the selected academic period is presented.

2 Estudiante de último semestre del programa de Administración de Empresas de la Universidad de los Llanos
Keywords: Dropout, repetition, academic retention, SPADIES (Prevention System of Higher Education), AIS (Academic Information System at the University of the Llanos).

\section{INTRODUCCIÓN:}

El Gobierno de Colombia, entendiendo que la Educación es el principal motor del desarrollo de los pueblos, se ha propuesto en los últimos años, como uno de sus principales objetivos, mejorar la calidad, la cobertura y la pertinencia de la educación en sus diferentes etapas: primaria, secundaria y superior. Para lograr tal cometido se han emitido una serie de leyes, decretos y políticas que han ayudado a cambiar la mentalidad y aunar esfuerzos con miras a subir los índices que dan cuenta de esas variables. No obstante lo anterior, sigue preocupando a la comunidad académica que la calidad y la pertinencia no hayan permeado todas las estructuras tal y como se esperaba.

Algo similar sucede con el tema de la cobertura pues, aunque día a día son más los niños y jóvenes que tienen acceso a la educación, los niveles de deserción no han tenido una mejoría significativa. Y esto es más evidente en lo que atañe a la Educación Superior, ya que escasamente el $50 \%$ de los estudiantes que ingresan a este sistema concluyen su carrera. Según estadísticas del Ministerio de Educación nacional, de cada cien estudiantes que ingresan a una institución de Educación Superior, cerca de la mitad no logra terminar su ciclo académico y obtener su título profesional. De ahí que el tema de la deserción ha tomado especial importancia, no sólo para el Estado colombiano sino también para las instituciones educativas, pues aunque se haya incrementado el número de matriculados, los niveles de deserción y de repitencia académica no han disminuido como se esperaba,

Teniendo en cuenta lo anterior, la facultad de Administración de Empresas de la Universidad de los Llanos, se ha propuesto como uno de los proyectos de su plan de mejoramiento, la elaboración de un estudio de deserción y repitencia académica que dé cuenta de estos dos fenómenos en el programa, durante el período 2008 - 2012, con la intención de proponer acciones concretas que ayuden a su 
disminución. Así las cosas, el estudio que se llevó a cabo, buscaba diagnosticar y analizar los factores determinantes de la deserción universitaria y la repitencia académica en el programa en mención y proponer estrategias que de manera articulada, ayudasen a la retención estudiantil y al mejoramiento del rendimiento académico de sus estudiantes.

El presente artículo ofrece al lector una visión sucinta de los resultados obtenidos en este estudio. Para lograr tal cometido se ha dividido el mismo en dos partes: En la primera se expone, de manera resumida, el marco conceptual y la metodología utilizada; en la segunda se presenta una síntesis del diagnóstico de la deserción y la repitencia académica del programa en el periodo seleccionado, el cual permitió hacer un análisis de sus causas y una caracterización de la población en riesgo académico

\section{CONCEPTUALIZACIÓN Y METODOLOGÍA DEL ESTUDIO}

Son dos los conceptos principales que el estudio, objeto de este artículo, abordó: la deserción y la repitencia académica. La primera la entendió como aquella situación a la que se enfrenta un estudiante cuando aspira y no logra concluir su proyecto educativo, considerándose como desertor a aquel individuo que, siendo estudiante de una institución de educación superior, no presenta actividad académica durante dos semestres académicos consecutivos, lo cual equivale a un año de inactividad académica. En algunas investigaciones este comportamiento se denomina como "primera deserción", ya que no se puede establecer si pasado este período el individuo retomará o no sus estudios o si decidirá iniciar otro programa académico. Esta es la definición que ha adoptado el Ministerio de Educación Nacional para la medición y seguimiento de la problemática.

Teniendo en cuenta esta definición, se puede diferenciar dos tipos de abandono en estudiantes universitarios: uno con respecto al tiempo y otro con respecto al espacio. En relación con el tiempo, la deserción puede ser precoz (individuo que habiendo sido admitido por la institución no se matricula), temprana (individuo que abandona sus estudios en los primeros semestres del programa) o tardía (individuo que abandona los estudios en los últimos semestres). Y respecto al espació, la deserción se divide en: institucional, cuando el estudiante abandona la universidad, o interna o del programa académico, cuando el estudiante se cambia de programa y se transfiere a otro que ofrece la institución.

La deserción estudiantil, independientemente de las condiciones y modalidades de presencialidad, es decisión personal del sujeto y no obedece a un retiro académico forzoso (por el no éxito del estudiante en el rendimiento académico, como es el caso de expulsión por bajo promedio académico) o el retiro por asuntos disciplinares. Diríase entonces que la deserción es opción del estudiante, influenciado positiva o negativamente por circunstancias internas o externas. De ahí que sea preciso diferenciar entre deserción (y variables asociadas), de mortalidad estudiantil, dado que la primera es intrasujeto y la segunda es extrasujeto.

Por otro lado, se entiende la repitencia como la acción de cursar reiterativamente una asignatura, sea por mal rendimiento del estudiante o por causas ajenas al ámbito académico. La repitencia se refleja en el atraso o rezago escolar, es decir, en la prolongación de los estudios por sobre lo establecido formalmente para cada carrera o programa. ${ }^{3}$

Teniendo en cuenta estos dos fenómenos que afectan la retención estudiantil "el Ministerio de Educación Nacional de Colombia, con apoyo del Centro de Estudios sobre Desarrollo Económico (CEDE) de la Universidad de los Andes, diseñó y desarrolló una metodología y herramienta que permite la observación permanente de un estudiante cuando esté matriculado para identificar aquéllos que presentan una mayor probabilidad de suspender temporal o definitivamente sus estudios, tomar medidas preventivas y mantener una información más precisa sobre la deserción estudiantil. Este programa llamado Sistema de Prevención de la Deserción en Educación Superior -SPADIES- es una herramienta informática que permite a las instituciones y al sector hacer seguimiento a la deserción estudiantil, identificar y ponderar variables asociadas al fenómeno, calcular el riesgo de deserción de cada estudiante a partir de condiciones académicas y socioeconómicas, y facilitar la elección, seguimiento y evaluación de impacto de estrategias orientadas a

3 INSTITUTO INTERNACIONAL PARA LA EDUCACIÓN SUPERIOR EN AMÉRICA LATINA Y EL CARIBE. Estudio sobre la Repitencia y Deserción en la Educación Superior en Guatemala, CALDERÓN DÍAZ; José Humberto: Guatemala de la Asunción, 2005. p. 13. 
disminuirlo. El estudio y tratamiento de la deserción estudiantil permite apoyar a cada estudiante según su situación específica, logrando una intervención más pertinente, oportuna y con mejores resultados.

Con base en la información que provee el SPADIES el Ministerio ha dinamizado el Fondo de Bienestar Universitario para apoyar el fortalecimiento de programas de retención estudiantil en instituciones de educación superior y a partir de 2008 a aquéllas con mayores niveles de deserción estudiantil, dadas las vulnerabilidades académicas y socioeconómicas de sus estudiantes. Con la implementación de estas acciones, el Ministerio de Educación Nacional espera avanzar en la principal meta que tiene prevista el país en la materia: disminuir la deserción por cohorte al $40 \%$ en 2010 y al $25 \%$ en $2019 " 4$.

Así mismo muchas universidades han diseñado, elaborado e implementado diversos planes, estrategias y herramientas para atacar la problemática aquí abordada. Universidades como la Nacional, Antioquia, Atlántico, Unillanos, Santo Tomás, Andes, Javeriana, Bolivariana, entre otras, tienen dentro de sus programas bandera sendos planes que, articulando la academia, la administración, el bienestar universitario y la familia han arrojado resultados significativos, dignos de imitar.

Por su parte, el programa de Administración de Empresas de la Universidad de los Llanos, siguiendo los derroteros emanados por la Alta Dirección y en concreto por el PREU (Programa de Retención Estudiantil Unillanista), ha planteado dentro de su plan de mejora la realización de un diagnóstico que dé cuenta de esta realidad al interior de la carrera. Es así como se llevó a cabo este estudio de tipo descriptivo, que hoy se presenta ante la comunidad académica y el cual tuvo como población objeto los desertores del programa durante los años 2008 y 2012 y como fuente de información el SPADIES, el SIA (Sistema de Información Académico de la Universidad de los Llanos) y los datos aportados por una encuesta hecha a un número importante de estos desertores.

Los sistemas de información del cual se tomaron los primeros datos contempló las variables que a continuación se relacionan y cuyos resultados

4 MINISTERIO DE EDUCACIÓN NACIONAL, Deserción Estudiantil en la Educación Superior Colombiana, Introducción, Bogotá: 2009. p. 17. permitieron hacer una caracterización de los estudiantes desertores y por ende ofrecer pistas acerca de los estudiantes en riesgo: distribución porcentual del último semestre que estudiaron en la Unillanos los estudiantes desertores, distribución porcentual del año y período del estudio de los estudiantes desertores, distribución porcentual de los desertores según género, distribución porcentual de la edad de los desertores, distribución porcentual del estado civil de los estudiantes desertores, distribución porcentual del tipo de colegio donde estudió el bachillerato el desertor, distribución porcentual de cómo se sostenía económicamente el desertor, distribución porcentual de cómo se sostenía económicamente el desertor, distribución porcentual de las causas que motivaron al desertor a no continuar en la Unillanos, distribución porcentual de la mayor dificultad en relación con la formación profesional de los desertores, distribución porcentual de si el desertor está estudiando actualmente, repitencia del programa administración de empresas por áreas de formación profesional, repitencia del programa administración de empresas por área de formación profesional, repitencia del programa Administración de Empresas por área de formación básica, repitencia del programa por área de formación humanística, repitencia del programa por semestres, asignaturas con mayor repitencia de los períodos entre 2008-1 al 2012-2.

\section{DIAGNÓSTICO DE LA RETENCIÓN Y LA REPITENCIA ACADÉMICA}

De acuerdo con las variables que el estudio tuvo en cuenta y aplicados los respectivos instrumentos, se obtuvieron los siguientes resultados y conclusiones:

- La mayoría de los estudiantes encuestados presentan una deserción temprana, pues el $36 \%$ manifiestan haberse retirado en el primer semestre y el $22 \%$ en el cuarto, lo cual sumado a los porcentajes del segundo (3\%) y tercer semestre (14\%) evidencian que más del $50 \%$ de los estudiantes desertores lo hacen antes de la mitad de la carrera.

- El mayor porcentaje de deserción se da entre los hombres con un $56 \%$, mientras que en las mujeres ese porcentaje es del $44 \%$.

- En cuanto a la edad de quienes decidieron retirarse del programa se encontró que el mayor porcentaje corresponde a aquellos que se ubican entre los $21 \mathrm{y}$ los 25 años con un $53 \%$, seguido de los que cuentan 
con edades que oscilan entre los 26 y los 30 años con un $22 \%$, lo cual es apenas lógico, teniendo en cuenta que estas son las edades normales del ciclo de estudios universitarios. No obstante lo anterior, llama la atención que un $17 \%$ de los desertores son menores de 20 años, lo que quiere decir que es importante hacer un mayor acompañamiento a los más jóvenes para evitar su pronto retiro.

- El estado civil de los estudiantes no parece ser una causa determinante de la deserción pues sólo el $17 \%$ de los desertores manifestaron tener un compromiso marital estable que les impidió continuar sus estudios y el $83 \%$ restante indicaron no tener ningún tipo de compromiso conyugal.

- El $86 \%$ de los desertores informaron que provenían de colegios públicos y tan solo $14 \%$ se graduaron en colegios privados. Muy posiblemente esta variable esté ligada a los recursos económicos con que cuenta la familia para apoyar los estudios de sus hijos, ya que quienes han realizado su bachillerato en colegios privados provienen en su mayoría de estratos cuyos padres asumen todos los gastos de manutención y estudio.

- El porcentaje de desertores que al ingresar a la universidad se sostenían con ingresos propios corresponde a $53 \%$, mientras que el $47 \%$ dependían económicamente de sus padres. Es de suponer que los ingresos de los que debían costearse sus estudios dependían básicamente de lo obtenido por su trabajo, lo que quiere decir que éstos debían laborar para poder estudiar. Cruzando estas dos últimas variables se ratifica lo que se afirmaba en el anterior párrafo, que las posibilidades de desertar aumentan a medida que el estudiante debe asumir los gastos de su manutención para continuar con sus estudios.

- Entre las causas que motivaron la decisión de no seguir estudiando en el programa, los estudiantes desertores ubicaron como la mayor causa la dificultad económica con el $28 \%$. Le siguen en orden de recurrencia: el bajo rendimiento académico con el $18 \%$, la ubicación laboral con un $15 \%$, cambio de carrera y de ciudad con un $10 \%$ cada una y finalmente con el $3 \%$ cada uno otras variables como el aplazamiento de semestre y la enfermedad.

- El $36 \%$ de los estudiantes desertores manifestaron que el manejo del tiempo libre fue la mayor dificultad que se les presentó, seguida por "otra" con un $31 \%$, entre las que se ubicaron motivaciones tales como: "el programa no cumplió las expectativas, "malas relaciones con los docentes y/o compañeros", "no les gusto la metodología", "no querían estudiar esa carrera", etc. con el (31\%).

- El estudio arrojó que un $53 \%$ de los desertores manifestaron que si continuaron la etapa de formación y un $47 \%$ que no. De los que si siguieron estudiando, la mayoría se encuentra adelantando carreras afines en otras instituciones o institutos como el SENA.

- El 33\% de quienes tenían más de 30 años de edad tuvieron como principal causa de deserción el cambio de ciudad, mientras que otro $33 \%$ lo hizo por bajo rendimiento, e igual porcentaje en esa edad, tuvo como razón de retiro el impacto que le generó el modelo de proyecto educativo.

- El 50\% de quienes se encontraban entre 15 y 20 años de edad desertaron por dificultades económicas, y el 33\% de los estudiantes entre 21 y 25 años también lo hicieron por esta misma razón. De otro lado, el $43 \%$ de los estudiantes entre los 26 y 30 años de edad, se retiraron porque cambiaron del lugar donde se encontraban laborando.

- De acuerdo al género se encontró que el $40 \%$ de las mujeres manifestó que desistió de estudiar en la Universidad principalmente por tener dificultades económicas. Para el caso de los hombres, la principal razón de deserción obedece al bajo rendimiento (23\%), las dificultades económicas (23\%), y la ubicación laboral (18\%). Se observa entonces que son las mujeres quienes, a causa de problemas económicos se ven en la obligación de desistir de continuar su formación profesional, situación que puede interpretarse como desventajoso para la mujer que desea profesionalizarse y vincularse posteriormente y con mejores condiciones al mercado laboral.

- Como se ha indicado anteriormente, una de las principales causas de la deserción tanto en hombres como mujeres (23\% y $40 \%$ respectivamente) son las dificultades económicas, y esto se explica en razón a la situación particular en torno a la fuente de los recursos de sostenimiento la cual, tanto para hombres como para mujeres, son los ingresos propios obtenidos por actividades laborales que desempeñan los estudiantes en horas extra clase y jornadas contrarias. El $50 \%$ tanto de hombres como de| mujeres se sostienen por recursos propios, lo que 
explica el impacto de las dificultades económicas en la consecución y culminación de los estudios universitarios.

- El $40 \%$ de quienes se sostienen por ingresos propios desertaron principalmente por las dificultades económicas, mientras que para los que tenían algún tipo de ayuda de padres o familiares, la principal causa de deserción correspondió al bajo rendimiento. Es decir que una vez superada la causa por motivos económicos, la segunda razón de deserción es el bajo rendimiento que presentan los estudiantes.

- Esta investigación encontró que en los estudiantes desertores mayores de 30 años el manejo de las matemáticas es la mayor dificultad para sostenerse en los estudios universitarios. Por su parte se evidenció que en los estudiantes de 26 a 30 años, la mayor dificultad obedece al manejo del tiempo libre (71\%).

- Quienes se encuentran entre 15 y 20 años, presentan como principal dificultad los altos niveles de exigencia con $50 \%$, mientras que los que se encuentra entre los 21 y los 25 años de edad, les afecta en un $38 \%$ tanto el manejo de las matemáticas como el manejo del tiempo libre.

- De acuerdo al género, el tema del manejo de las matemáticas afecta más a los hombres que a las mujeres (40\% y 20\%, respectivamente), siendo contrario en el caso del manejo del tiempo libre, donde el $53 \%$ de las mujeres lo presentan como principal dificultad frente apenas el $27 \%$ de los hombres. Lo anterior permite establecer que las mujeres universitarias de Administración de Empresas en el período estudiado tienen un mejor nivel de matemáticas que les permite afrontar mejor la carga académica en estas áreas frente a los hombres.

- El 34\% de los estudiantes que provienen de colegios públicos son quienes afrontan mayores problemas en el manejo de las matemáticas (34\%), frente a un $20 \%$ de los que vienen de colegios privados. Así mismo, los altos niveles de exigencia académica son un problema para los estudiantes provenientes de establecimiento públicos (19\%), mientras que ninguno de los estudiantes que provienen de los colegios privados sostiene que éste sea un problema. Sin embargo, se observa que el $40 \%$ de quienes provienen de instituciones privadas tienen dificultades a la hora de relacionarse con los compañeros, lo cual puede entenderse como una respuesta normal a la diferencia entre los ambientes y la convivencia en las Universidades públicas y las privadas.

- El área con mayor repitencia en el Programa de Administración de Empresas de la Universidad de los Llanos, según información suministrada por el Sistema de Información Académica de la institución, es el área de Formación Profesional con un $60 \%$, seguida del área de Formación Humanística con un $25 \%$ y por último el área de Formación Básica con un $15 \%$.

- En cuanto al Área de Formación Profesional se encontró que los estudiantes reprueban con mayor porcentaje las asignaturas de Economía y Finanzas con un $58 \%$, seguido de las de Administración y Organizaciones con un porcentaje del $35 \%$, mientras que en las asignaturas de Mercadeo, Gerencia de Producción e Investigación de Operaciones los estudiantes las reprueban con un $4 \%$ y un $3 \%$ respectivamente.

- Para el Área de Formación Básica se comprobó una repitencia constante en las asignaturas de Estadística I y II y Matemáticas I y II. Así por ejemplo, durante el periodo estudiado, se pudo evidenciar que: de 320 estudiantes que cursaron Estadística II, 57 la perdieron, de 319 que adelantaron Estadística I, 51 la reprobaron, de 306 que matricularon Matemáticas II, 87 no pudieron pasarla y de 359 que estudiaron Matemáticas I, 83 tuvieron que repetirla. Los factores para la repitencia de estas materias son varios, entre los que se destacan: la predisposición con base en las experiencias que han tenido los estudiantes en relación con las asignaturas, la metodología de enseñanza del docente, errores que comete el estudiante al aplicar los conceptos, débil formación desde la educación media y la media básica entre otros.

- En el área de Formación Humanística las materias que más reprueban los estudiantes son las de los primeros semestres, tales como Derecho Constitucional, de segundo semestre, que de 414 estudiantes que tomaron la asignatura, 90 la perdieron; Pensamiento Lógico Matemático, de primer semestre, que de 368 estudiantes la reprobaron 74; y Derecho Comercial, de tercer semestre, con 70 reprobados de 320 que la cursaron. Llama la atención que de este grupo, son las materias 
de área del derecho las que presentan un alto porcentaje de repitencia.

- El estudio mostró que el fenómeno de la repitencia va disminuyendo conforme aumenta el semestre que cursan los estudiantes. Es así como entre el primer y quinto semestre, el número de estudiantes que pierden asignaturas es mayor que el de grados superiores. Los estudiantes pierden menos asignaturas una vez superan quinto semestre, se presume que aumenta el compromiso del estudiante con respecto a su rendimiento académico y que se ha logrado la asimilación de los conceptos básicos del programa.

- El estudio mostró que el fenómeno de la repitencia va disminuyendo conforme aumenta el semestre que cursan los estudiantes. Es así como entre el primer y quinto semestre, el número de estudiantes que pierden asignaturas es mayor que el de grados superiores. Los estudiantes pierden menos asignaturas una vez superan quinto semestre, se presume que aumenta el compromiso del estudiante con respecto a su rendimiento académico y que se ha logrado la asimilación de los conceptos básicos del programa.

- Se pudo constatar que algunas de las causas de la repitencia en estos primeros semestres fueron, entre otras: la falta de orientación vocacional, la dificultades de adaptación a la universidad, ya sea por razones de edad o de procedencia pues muchos de los estudiantes de la Unillanos provienen de otras regiones y se les hace difícil adecuarse a otra ciudad y al ritmo universitario, con el agravante de estar lejos de sus hogares.

- Teniendo en cuenta que muchas de las causas de la deserción y la repitencia académica de los estudiantes de Administración de Empresas de la Universidad de los Llanos, tal y como lo evidencia este estudio, no son sólo de carácter académico, sino que provienen de factores diferentes (económicos, psicológicos, físicos, sociales, etc.) se requiere implementar estrategias que apoyen la formación desde otros ámbitos y disciplinas y mitigar de esta manera los efectos negativos de estas variables. Dentro de estas estrategias se pueden mencionar, entre otras, las siguientes: Diagnóstico inicial del estudiante al ingresar al programa, elaboración de un plan de acompañamiento desde los ámbitos que así lo requieran, tutorías académicas, orientación vocacional, asesoría psicológica, terapias familiares y de pareja, atención médica especializada, guardería infantil para los hijos de los estudiantes, cursos de nivelación, talleres de apoyo (técnicas de estudio y lectura), becas académicas y socioeconómicas, auxilios económicos para el sostenimiento del estudiante (alimentación, útiles de estudio, etc.), bolsa de empleo y finalmente la incorporación de programa al observatorio MAUTE para hacer un seguimiento permanente y estricto del desempeño académico de los estudiantes, especialmente de aquellos que están en riesgo de deserción.

\section{CONCLUSIONES}

- El fenómeno de la deserción académica de los estudiantes del programa de Administración de Empresas de la Universidad de los Llanos está influenciado por múltiples factores, tanto de orden interno como de orden externo, que condicionan fuertemente la decisión de los alumnos de retirarse de sus estudios.

Dentro de estos se puede mencionar: Factores psicosociales (problemas de aprendizaje, dificultades en las relaciones interpersonales y familiares, falta de habilidades y competencias sociales, problemas o enfermedades mentales -depresión, baja autoestima, consumo de sustancias psicoactivas- y físicas que puedan dificultar el aprendizaje); factores motivacionales o vocacionales (falta de claridad en las motivaciones o desconocimiento de la carrera o profesión y presiones familiares o de amistadas para cursar el programa), factores académicos (bajo rendimiento académico, falta de bases en las áreas básicas, problemas de competencias comunicativas, de comprensión lectora $\mathrm{y}$ de escritura $\mathrm{y}$ de aprendizaje); factores económicos (escases de recursos económicos, necesidad de trabajar para su sostenimiento, compromisos conyugales o de paternidad, falta de apoyo por parte de los padres, etc); factores Institucionales (ambiente universitario desfavorable, desconocimiento de procedimientos y reglamentos, baja calidad académica, docentes con bajas condiciones académicas y pedagógicas, relaciones interpersonales tensas con docentes, directivos, administrativos y compañeros de estudio, etc.

- A partir de los datos arrojados por el estudio se descubrió que la tasa de deserción es mayor en los hombres que en las mujeres; que los estudiantes con bajos y medios resultados en el Examen de Estado presentan mayor tasa de deserción que aquellos con resultados altos; que los estudiantes que trabajaban al 
presentar el ICFES muestran una tasa de deserción más alta que los que no trabajaban; que los apoyos académicos y financieros ayudan efectivamente a disminuir la tasa de deserción y entre más apoyos esta tasa se hace menor; que la tasa de deserción por cohorte acumulada aumenta hasta el sexto semestre, donde se estabiliza y finalmente, entre otros aspectos, que en promedio entre los años 2008 y 2012 la tasa de deserción por período es $10.33 \%$.

- En cuanto a repitencia se refiere, se pudo constatar que ésta disminuye a medida que aumenta el semestre que cursan los estudiantes, lo cual se evidencia en el hecho de que entre el primer y quinto semestre es donde se presenta mayor pérdida de asignaturas y una vez cursado el quinto semestre hay mayor supervivencia y menos perdidas de asignaturas en el programa. Así mismo se encontró que las materias con mayor repitencia son: Derecho Constitucional con un porcentaje del (23\%), seguido de Matemáticas I (23\%), Matemáticas Financieras con (19\%), Gestión del Talento Humano (19\%) y finalmente Derecho Comercial con $(18 \%)$.

- Toda vez que varias de las causas de la deserción y la repitencia académica estudiantil en el programa de Administración de Empresas de la Unillanos, no son sólo de carácter académico, sino también de otra índole (económica, psicológica, física, social, etc.) se requiere implementar estrategias que, desde diferentes ámbitos y disciplinas, apoyen la formación universitaria y mitiguen los efectos negativos de estas variables.

\section{BIBLIOGRAFÍA}

INSTITUTO INTERNACIONAL PARA LA EDUCACIÓN SUPERIOR EN AMÉRICA LATINA Y EL CARIBE. Estudio sobre la Repitencia y Deserción en la Educación Superior en Guatemala, CALDERÓN DÍAZ; José Humberto: Guatemala de la Asunción, 2005. p. 13.

MINISTERIO DE EDUCACIÓN NACIONAL, Deserción Estudiantil en la Educación Superior Colombiana, Introducción, Bogotá: 2009. p. 17.

MINISTERIO DE EDUCACIÓN NACIONAL. Deserción estudiantil en la educación superior, metodología de seguimiento, diagnóstico y elementos para su prevención. El estudio de la deserción estudiantil en Colombia. Bogotá: MEN. 2009. p. 35.
MINISTERIO DE EDUCACIÓN NACIONAL. Deserción estudiantil en la educación superior, metodología de seguimiento, diagnóstico y elementos para su prevención. Teorías sobre la deserción estudiantil. Bogotá: MEN. 2009. p. 20.

MINISTERIO DE EDUCACIÓN NACIONAL. Deserción estudiantil en la educación superior, metodología de seguimiento, diagnóstico y elementos para su prevención. Una introducción a los modelos de duración para el análisis de la deserción estudiantil. Bogotá: MEN. 2009. p. 40.

REVISTA UNIVERSIDAD EAFIT. PARAMO, Gabriel Jaime y CORREA, Carlos Arturo. Deserción Estudiantil Universitaria. Conceptualización: EAFIT, 1999.

SISTEMA PARA LA PREVENCIÓN DE LA DESERCIÓN DE LA EDUCACIÓN SUPERIOR SPADIES. Disponible en Internet: http://www.mineducacion.gov.co/sistemasdeinforma cion/1735/w3-article-254668.html

UNIVERSIDAD DE LOS LLANOS, Estudio de la Deserción Estudiantil de los programas de pregrado de la Universidad de los Llanos 1998- 2004, MALAGÓN ESCOBAR, Luz; CALDERÓN CAÑON, Cesar Augusto y SOTO HERNÁNDEZ, Edwin. Villavicencio: 2006, p. 32.

UNIVERSIDAD DE LOS LLANOS. Quienes Somos. Disponible en Internet: http://web.unillanos.edu.co/index.php/quienessomos/mision-vision-objetivos.html

UNIVERSIDAD DE LOS LLANOS. Programa de Retención Estudiantil Unillanista - 2012. Disponible en Internet: 